\title{
Optimum Planning Use of Equipment in Agriculture
}

\author{
Rishat Zaynagabdinov $^{1}$ (D), Ildar Gabitov ${ }^{1}$ iD Ilshat Bakiev $^{1}$ (iD), Ildar Gafurov ${ }^{1}$ iD, Konstantin Kostarev $^{1}$ i \\ ${ }^{1}$ Federal State Budgetary Educational Establishment of Higher Education. Bashkir State Agrarian University (Russian Federation) \\ zaynagabdinovri@rambler.ru,gabitovil@rambler.ru,bakievils@rambler.ru,gafurovil@rambler.ru,kostarevko@rambler.ru
}

Received: April 2020

Accepted: July 2020

\section{Abstract:}

Purpose: to reduce operating costs when performing agricultural mechanized work.

Design/methodology/approach: The development of the desired economic and mathematical model of the optimal MTA distribution by type of work is based on the above condition it is necessary to plan the work of not tractor brands indicating their number, but each tractor individually.

Findings: It is known that the availability of equipment varies during the season, so it was studied in dynamics. In this regard, to solve this problem, neural-network simulation was first used. As a result of the search, a network of the type of radial basis function was obtained that best describes these dependencies

Research limitations/implications: the authors developed a fundamentally new mathematical model for optimizing the use of technology, which, unlike existing ones, allows to:

- $\quad$ plan the operation of each tractor individually;

- designate the so-called "mandatory tractors", that is, those that the user, at his discretion, would like to assign to a particular operation or vice versa, would like the tractor operator not to work on this operation.

Originality/value: Thus, the scientific novelty of the work performed are:

- a technique for operational planning of work and increasing the efficiency of using the MTA, taking into account the technical condition of the tractors, the specific conditions for their functioning and the work experience of the machine operator.

- a mathematical model for solving the problem of the optimal distribution of MTA by type of work and the mathematical apparatus for its implementation;

- a technique for adjusting the performance of the MTA, taking into account the experience of the tractor driver, the service life and technical readiness of the tractor.

Keywords: agriculture, DT-75, and T-402 tractors, optimal plan, productivity and fuel consumption, integer programming, reduction of terms, and costs

\section{To cite this article:}

Zaynagabdinov, R., Gabitov, I., Bakiev, I., Gafurov, I, \& Kostarev, K. (2020). Optimum planning use of equipment in agriculture. Journal of Industrial Engineering and Management, 13(3), 514-528 https://doi.org/10.3926/jiem.3185 


\section{Introduction}

Optimal planning of equipment use is one of the most important reserves for increasing the efficiency of agricultural production. Since the operating costs of the machine and tractor fleet in the structure of the cost of agricultural products reach $30-40 \%$, due to the optimal distribution of tractors, it is possible to reduce the cost of the work significantly, to minimize the duration of their implementation. It does not require investment; therefore, in the face of a shortage of resources, the solution of optimal planning problems is very relevant.

In scientific works, the problem of optimal planning of the use of technology is often reduced to the task of a salesman (Wu, Weise \& Chiong, 2015; Lin, Sun \& Salous, 2016). Tractors and agricultural machinery as a resource are present in the tasks of the so-called maximin, where the optimization criterion is maximized or minimized depending on the purpose (Recht, 2016).

In Parwanto, Morohosi and Oyama (2015), the search for an optimal plan for the equipment used is reduced to solving the problem of optimizing traffic flow. In most cases, the solution to the problem is a non-integer, and the data obtained must be rounded. As a result, the optimality of the plan is violated, as well as the consistency of technologically related operations. A method of a partial solution to this problem is proposed in work, where a mixed integer programming algorithm is used (Luo, 2019). However, this method is complicated for practical use in the distribution of much equipment; then, there are solutions to problems of immense dimensions.

When constructing optimal planning models, not all researchers take into account the need for additional time for transferring M.T.A. from one field to another and for re-aggregation. For real conditions, this is unacceptable. In Wu, Zhou, Qiao and Wang. (2015), it is proposed to solve this problem using separate temporal and spatial planning.

Most of the proposed models initially assume that tractors and agricultural machines are located in one single point and are returned to this point every day at the end of work. However, in production, there may be cases when tractors are based at various points. In this case, it is necessary to introduce additional conditions into the model that take into account such a territorial spread of the bases. This item is taken into account in Allate (2019).

Indicators of the use of equipment will vary significantly depending on the adopted objective function. For example, while minimizing the timing of agricultural operations, which is very important for a probable change in weather conditions, fuel consumption can be up to 1.5 times higher than with an optimal distribution according to the criterion of minimum fuel consumption (Zhao, 2017). Only through mathematical optimization calculations can you choose the best plan from hundreds of possible ones.

In Shevtsov, Lavrov and Izmailov (2015), the task of operational planning of the equipment used is reduced to the problem of optimizing the composition of the machine-tractor fleet (M.T.F.). Unusual methods for solving the problem in a network formulation (Jourquin, 2018). But such models are difficult to formalize.

Questions linking the planning of M.T.A. work and a more accurate determination of their characteristics (optimal operating modes, fuel consumption, productivity) were considered in Arzhenovskiy (2017), Bulgakov, Kyurchev, Nadykto, Beloev, Kangalov and Mitev (2017).

In Bulgakov et al. (2017), a new method for classifying tractors was proposed - not by traction power (as is done in most countries of the world), but by the nominal traction developed by them. The author notes that this will allow for more accurate calculations when aggregating various agricultural machines, increasing their overall level of productivity. However, the use of tractor classification is necessary only for cases of integrated planning, and most often, in solving the problem of forming the optimal composition of the fleet, and not their operational distribution.

In Yakovenko, Makarchuk and Serbinov (2019), the issue of the most rational manning of agricultural machines and tractors is considered to obtain higher unit productivity with lower fuel consumption. However, the issue is considered in isolation from the further distribution of these units by the type of work. 
The range of mathematical methods used to optimize the distribution of aggregates by type of work is quite extensive. It is due to differences in the description of production processes. The simplex method was the most widely used (Rüttimann, 2015; Tanaka, 2019).

Modern researchers also propose modified linear programming algorithms that either formally simplify (Belahcene, Marthon \& Aidene, 2018) or speed up the solution of the problem (Bibi \& Bentobache, 2015).

In Wan and Wei (2019), a model is proposed for selecting the best plan for using the technique for the case of multicriteria multimodal resource allocation based on a hybrid algorithm. However, in practice, such a multichip statement of the problem is extremely rare.

As mentioned above, the authors neglect the requirement of integrality in solving practical problems, explaining that it is challenging to solve problems of such colossal dimensions without taking this requirement into account. Therefore, if the problem touches upon the problem of integer variables, then the apparatus of partially integer programming is used.

However, despite the variety of mathematical models for optimizing the M.T.A. work plan, all of them are not sufficiently adapted for their practical use in the conditions of conventional farms. They have not received the actual application (Wan \& Wei, 2019). The reason is as follows.

In real production, productivity and fuel consumption by tractors, even within the same brand, are not the same. It is due to the difference in the conditions of their work, the service life of the equipment, qualifications, and length of service of machine operators. That is, even the same compositions M.T.A. has different performance indicators (performance, fuel consumption). The actual performance of M.T.A. more than two times may differ from the normative. As a result, the plan drawn up can be not only non-optimal but also unrealizable. Therefore, each tractor must be distributed separately by type of work - and this is an optimization problem with Boolean variables, for which the existing economic and mathematical models are not suitable (Wan \& Wei, 2019). Also, this, in turn, dramatically increases the dimension of the problem, for which the existing mathematical apparatus is also not suitable., it is necessary to create unique methods, such as cutting planes and branches and borders, to solve discrete optimization problems. Still, they are extremely difficult for software implementation.

The study aims to reduce costs when performing M.T.A. agricultural mechanized work.

The following tasks were solved to achieve this goal:

1. To develop a mathematical model of the optimal distribution of M.T.A. by type of work, allowing you to plan the work of each unit of equipment separately.

2. Establish dependencies to account for the impact of tractor life and other factors on the performance of the M.T.A.

3. To develop a mathematical apparatus for solving the optimization problem with Boolean variables about the distribution of M.T.A. by type of work.

\section{Methods}

The development of the desired economic and mathematical model of the optimal M.T.A. distribution by type of work is based on the above condition - it is necessary to plan the work of not tractor brands indicating their number, but each tractor individually. The mathematical part of the model was developed by the author and described in Zainagabdinov (2004). In a busy period of mechanized fieldwork, when up to 15-20, different jobs are performed simultaneously, the most relevant criterion is the minimum total duration of operations. The objective function will look like this:

$$
\sum_{i=1}^{I} D_{i} \rightarrow \min
$$


The duration of the $\mathrm{i}$-th operation is:

$$
D_{i}=\frac{W_{i}}{w_{1 i} \cdot X_{1 i}+w_{2 i} \cdot X_{2 i}+w_{3 i} \cdot X_{3 i}+\ldots+w_{n i} \cdot X_{n i}}=\frac{W_{i}}{\sum_{n=1}^{N} w_{n i} \cdot X_{n i}}
$$

where $W_{i}$ - is the volume of work on the $i$ - th operation, ha;

$w_{n i}$ - is daily productivity of the unit with the tractor of the $n$-th number (from now on the $n$-ith unit) in the $i$-th operation, ha

$X_{n i}$ - is the required number of $n$-units in the $i$-th operation, pcs.

$X_{n i}$ - can take only two values - 0 or 1.

The objective function (2) is linear fractional; to bring it to a linear dependence, we transform this expression by dividing the unit by it and denote:

$$
\frac{1}{D_{i}}=\frac{w_{1 i} \cdot X_{1 i}+w_{2 i} \cdot X_{2 i}+w_{3 i} \cdot X_{3 i}+\ldots+w_{n i} \cdot X_{n i}}{W_{i}}=d_{i}
$$

or in another way:

$$
\frac{1}{D_{i}}=\frac{w_{1 i}}{W_{i}} \cdot X_{1 i}+\frac{w_{2 i}}{W_{i}} \cdot X_{2 i}+\frac{w_{3 i}}{W_{i}} \cdot X_{3 i}+\ldots+\frac{w_{n i}}{W_{i}} \cdot X_{n i}=d_{i},
$$

Then the expression (1) will take the form:

$$
\sum_{i=1}^{I} d_{i} \rightarrow \max
$$

The condition for using each tractor no more than once is written in the form:

$$
\begin{aligned}
& X_{11}+X_{12}+X_{13}+\ldots+X_{1 i}=\sum_{i=1}^{I} X_{1 i} \leq 1 \\
& X_{21}+X_{22}+X_{23}+\ldots+X_{2 i}=\sum_{i=1}^{I} X_{2 i} \leq 1 \\
& X_{31}+X_{32}+X_{33}+\ldots+X_{3 i}=\sum_{i=1}^{I} X_{3 i} \leq 1 \\
& X_{n 1}+X_{n 2}+X_{n 3}+\ldots+X_{n i}=\sum_{i=1}^{I} X_{n i} \leq 1
\end{aligned}
$$

The number of used agricultural machines and couplings should not exceed their number in the park:

$$
\begin{gathered}
\sum_{i=1}^{I} \sum_{n=1}^{N} X_{n i} \cdot \lambda_{n i s} \leq N_{s} \\
\sum_{i=1}^{I} \sum_{n=1}^{N} X_{n i \mu} \leq N_{\mu}
\end{gathered}
$$


Where $N_{s}, N_{\mu}$ - are respectively the available number of agricultural machinery-implements of the $s$ th brand and coupling of the $\mu$ brand;

$\lambda_{\text {nis }}$ - is the number of agricultural machines of the $s$ th type in the composition of $n$ units on the $i$ th operation;

$X_{n i \mu}$ - is the number of $n$ units on the $i$ th operation containing the $\mu$ brand coupling.

The condition for the coordination of the timing of technologically interconnected operations:

$$
D_{v_{1}} \leq D_{v_{2}} \leq \ldots \leq D_{v_{k-1}} \leq D_{v_{k}}
$$

where $v$ is the index of interrelated operations;

$k$ - is the number of interrelated operations.

For example, pre-sowing cultivation, sowing, and rolling crops are included in one block of interconnected operations. Two indices mark these operations. The first index $v$ denotes the number of this block, and it is the same for these types of jobs (for example, $\nu=1$ ). The second - $k$ shows the order of work. In this case, for cultivation $k=1$, for sowing -2 , for rolling - 3 , that is, according to condition (9), the duration of operation with a lower number should not exceed the duration of operation with a higher number.

In this example, the condition must be met:

$D_{1_{1}} \leq D_{1_{2}} \leq D_{1_{3}}$

This expression should be represented as follows to enter a computer program:

$$
d_{v_{1}} \geq d_{v_{2}} \geq d_{v_{3}} \geq \ldots \geq d_{v_{k-1}} \geq d_{v_{k}}
$$

or in another way:

$$
\left\{\begin{array}{l}
d_{v_{1}}-d_{v_{2}} \geq 0 \\
d_{v_{2}}-d_{v_{3}} \geq 0 \\
\cdots \ldots \ldots \ldots \ldots \ldots \\
d_{v_{k-1}}-d_{v_{k}} \geq 0
\end{array}\right.
$$

The objective function providing a minimum of total fuel consumption in operations is written as:

$$
\sum_{i=1}^{I} Q_{i} \rightarrow \min
$$

where $Q_{i}$ is the fuel consumption at the $i$ th operation, $\mathrm{kg}$

With

$$
Q_{i}=\left(w_{1 i} \cdot q_{1 i} \cdot X_{1 i}+w_{2 i} \cdot q_{2 i} \cdot X_{1 i}+w_{3 i} \cdot q_{3 i} \cdot X_{3 i}+\ldots+w_{n i} \cdot q_{n i} \cdot X_{n i}\right) \cdot D_{i}
$$

where $q_{n i}$ is the rate of per hectare fuel consumption of the unit at the $i$ th operation, $\mathrm{kg} / \mathrm{ha}$.

Then the expression (13) will take the form:

$$
Q_{i}=\frac{w_{1 i} \cdot q_{1 i} \cdot X_{1 i}+w_{2 i} \cdot q_{2 i} \cdot X_{2 i}+w_{3 i} \cdot q_{3 i} \cdot X_{3 i}+\ldots+w_{n i} \cdot q_{n i} \cdot X_{n i}}{w_{1 i} \cdot X_{1 i}+w_{2 i} \cdot X_{2 i}+w_{3 i} \cdot X_{3 i}+\ldots+w_{n i} \cdot X_{n i}} \cdot W_{i}
$$

In expanded form, function (12) is written as follows: 


$$
\begin{aligned}
& \sum_{i=1}^{I} Q_{i}=\frac{w_{11} \cdot q_{11} \cdot X_{11}+w_{21} \cdot q_{21} \cdot X_{21}+w_{31} \cdot q_{31} \cdot X_{31}+\ldots+w_{n 1} \cdot q_{n 1} \cdot X_{n 1}}{w_{11} \cdot X_{11}+w_{21} \cdot X_{21}+w_{31} \cdot X_{31}+\ldots+w_{n 1} \cdot X_{n 1}} \cdot W_{1}+ \\
& +\frac{w_{12} \cdot q_{12} \cdot X_{12}+w_{22} \cdot q_{22} \cdot X_{22}+w_{32} \cdot q_{32} \cdot X_{32}+\ldots+w_{n 2} \cdot q_{n 2} \cdot X_{21}}{w_{12} \cdot X_{12}+w_{22} \cdot X_{22}+w_{32} \cdot X_{32}+\ldots+w_{n 2} \cdot X_{n 2}} \cdot W_{2}+ \\
& +\frac{w_{13} \cdot q_{13} \cdot X_{13}+w_{23} \cdot q_{23} \cdot X_{23}+w_{33} \cdot q_{33} \cdot X_{33}+\ldots+w_{n 3} \cdot q_{n 3} \cdot X_{n 3}}{w_{13} \cdot X_{13}+w_{23} \cdot X_{23}+w_{33} \cdot X_{33}+\ldots+w_{n 3} \cdot X_{n 3}} \cdot W_{3}+\ldots
\end{aligned}
$$

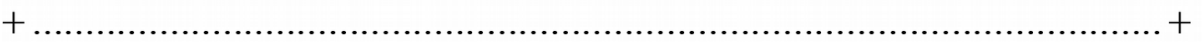

$$
\begin{aligned}
& +\frac{w_{1 i} \cdot q_{1 i} \cdot X_{1 i}+w_{2 i} \cdot q_{2 i} \cdot X_{2 i}+w_{3 i} \cdot q_{3 i} \cdot X_{3 i}+\ldots+w_{n i} \cdot q_{n i} \cdot X_{n i}}{w_{1 i} \cdot X_{1 i}+w_{2 i} \cdot X_{2 i}+w_{3 i} \cdot X_{3 i}+\ldots+w_{n i} \cdot X_{n i}} \cdot W_{i} \rightarrow \min
\end{aligned}
$$

The objective function of the minimum of the reduced costs will have a similar form, but instead of the $w_{n i} \cdot q_{n i}$ factors in the expression (15), there will be the values of the reduced costs when the $n$th unit performs the $i$ th operation.

The condition for the mandatory performance of the operation in an implicit form can be written in the following form:

$$
d_{j}>0
$$

An algorithm for integer optimization with Boolean variables based on the construction of a lexicographic sequence (L.S.) has been developed to solve this problem. The latter is used in the Balash method (Balas, 1967), which underlies most integer programming applications.

L.S. is an ordered table of zeros and ones as a plan for using available resources. For example, the following L.S. shows how theoretically three units can be distributed in different jobs.

\begin{tabular}{|c|c|c|c|}
\hline \multirow{2}{*}{$\begin{array}{c}\text { Series } \\
\text { number }\end{array}$} & \multicolumn{3}{|c|}{ Unit designation and use plan } \\
\cline { 2 - 4 } & $\mathbf{X} 1$ & $\mathbf{X} 2$ & $\mathbf{X} 3$ \\
\hline 1 & 0 & 0 & 0 \\
\hline 2 & 1 & 0 & 0 \\
\hline 3 & 0 & 1 & 0 \\
\hline 4 & 1 & 1 & 0 \\
\hline 5 & 0 & 0 & 1 \\
\hline 6 & 1 & 0 & 1 \\
\hline 7 & 0 & 1 & 1 \\
\hline 8 & 1 & 1 & 1 \\
\hline
\end{tabular}

Table 1. Lexicographic sequence for three variables

"0" in Table 1 means that the unit is not in use, and " 1 " is in use. The formula calculates the total number of distribution options:

$$
N=2^{p}
$$

where $\mathrm{p}$ is the number of distributed unit resources.

In our case, $p=3, N[(=2)]^{\wedge} 3=8$.

For each series, the target value is calculated, for example, costs. Next, the series are sorted by increasing (decreasing) this target value and the optimal one is selected from them. Optimization criteria allow us to exclude 
divergent series. For example, if $\mathrm{X} 1$ and $\mathrm{X} 2$ are units made up of the same tractor, then rows number 4 and 8 are excluded from consideration since the same tractor cannot be used simultaneously in two different jobs. Row number 1 with zeros only is also excluded, because it means that not a single unit is busy at work, that is, the work is not performed.

According to formula (17), the number of possible series grows in a power-law order, and the process of constructing an L.S. begins to become difficult many times over. For example, if $p=10$, then $N=1,024$. To build such a series without getting confused is almost impossible. A formula was derived for the formation of lexicographic sequence individual values, the value of a series $a$ member $(0$ or 1$)$ equals:

$$
a=\operatorname{int} \frac{n-1}{2^{k}-1}-\operatorname{int}\left[\frac{\operatorname{int} \frac{n-1}{2^{k}-1}}{2}\right] \times 2
$$

where $n$ is the series number;

$k$ - is the column number;

int - is a function to select the integer part of a number.

So the value of the variable is a function of the series number and column number. It allows us to significantly simplify the optimization program and reduce the time it takes to find a solution. For each operation, an L.S. block is compiled. Block formation allows us to deliberately exclude from consideration those points that would necessarily be analyzed when using the Balash method (Balas, 1967). The series are sorted in ascending order at minimization. And they are sorted in descending order at maximization. In the next step, combinations of compatible series are selected. The rows may be incompatible if the restrictions are not met and if the conditions for using each tractor are violated only once. The series enumeration program is designed so that the search for the optimum begins with the "best" options and moves to the "worst" ones until a satisfactory solution is obtained. All of the above mathematical parts are embedded in the computer program "Agromaster+", developed by the author. The program allows us to find the optimal solution using the method of truncated exhaustive search.

An essential feature of the model and mathematical apparatus proposed above is the possibility of assigning the so-called "mandatory" tractors. "Mandatory" refers to specific tractors that the user, at his discretion, would like to assign to a particular operation or, on the contrary, would like the tractor operator not to work on this operation. It is especially important since, on the ground, the final decision on the use of technology is made subjectively. The developed model allows subjective corrections to be laid in advance before solving the problem and to obtain the optimal feasible plan.

With an increase in tractor life, the performance of the unit decreases. One of the reasons for this is a decrease in the active power of its engine. Studies of Professor Plaksin (1975) found that this reduces losses in the transmission and due to slipping propulsors. Because of this, the actual traction efficiency of the tractor will be $2 \ldots . .5 \%$ higher than the calculated one. Using these, we derived a formula for adjusting the performance of the unit, taking into account the decrease in tractor engine power. To use it, you need to know how the value of the coefficient K_Ne from the operating time of the tractor changes. Subject to all amendments, the actual shift performance of the M.T.A. will be equal to:

$$
\begin{gathered}
\text { W rep f }=\mathrm{W} \mathrm{repr} \cdot \mathrm{K} \text { ter } \cdot \mathrm{K} \mathrm{rug} \cdot \mathrm{K} \text { rock } \cdot \mathrm{Kcc} \cdot \mathrm{K} \mathrm{h} \cdot \mathrm{K} \mathrm{tr} \times \\
\times(1,02 \ldots 1,05) \cdot\left(1-\frac{1-K_{N e}}{\mathrm{t} \mathrm{e}}\right) \cdot \mathrm{K} \mathrm{td} \cdot \mathrm{Kr} \cdot \mathrm{r}_{\mathrm{agrm}}^{\mathrm{n}}
\end{gathered}
$$

Where $\mathrm{W}$ rep $\mathrm{r}$ is the replaceable production rate of M.T.A. for healthy working conditions, ha; 
$\mathrm{K}$ ter, $\mathrm{K}$ rug, $\mathrm{K}$ rock, $\mathrm{Kcc}, \mathrm{K}$ h, $\mathrm{K}$ tr - are correction factors to the production rate, taking into account, respectively, the terrain, ruggedness by obstacles, rockiness, configuration complexity and field height above sea level, reduced traction on sandy soils (these coefficients are determined by known methods used to normalize mechanized work);

$\mathrm{K}$ td - is coefficient taking into account the impact of tractor driver qualifications on the performance of the unit;

$\mathrm{Kr}$ - is coefficient of technical readiness of the tractor;

$\mathrm{r}_{\mathrm{agrm}}^{\mathrm{n}}$ - is the coefficient of operational reliability of an agricultural machine;

$n$ - is the number of agricultural machinery in the unit;

$\mathrm{t}$ e - is traction efficiency of the tractor at rated engine power;

$K_{N e}=\frac{N e_{i}}{N_{e H}}$ - is coefficient taking into account the decrease in the sufficient engine power as the service life of the tractor increases, where

$N e_{i}$ - actual effective engine power of the tractor of the year of service, $\mathrm{kW}$;

$N e_{H}$ - rated engine power, $\mathrm{kW}$.

The factor $(1,02 \ldots 1,05) \cdot\left(1-\frac{1-K_{N e}}{\eta_{m н}}\right)$ in the formula (19) takes into account the decreasing effect in the sufficient engine power with an increase in the tractor's "age" on the performance of the unit.

Experimental studies were conducted in agricultural enterprises of the Republic of Bashkortostan to obtain the values of individual parameters included in the formula (19). The power efficiency of the caterpillar tractors $\Delta T-75$ and T-402 engines were measured before and after major repairs of the engines, and before and after their operational adjustment. For measurements, the ИМАЦ-M device was used.

To establish the dependence of tractor performance on the experience of tractor drivers, statistical data on the use of tractors in the farms of the Republic of Bashkortostan were analyzed, photo-timing observations were carried out. Preparation for observations and their implementation was carried out by the methodology of the State Scientific-Technical Institute.

\section{Research Results and Discussion}

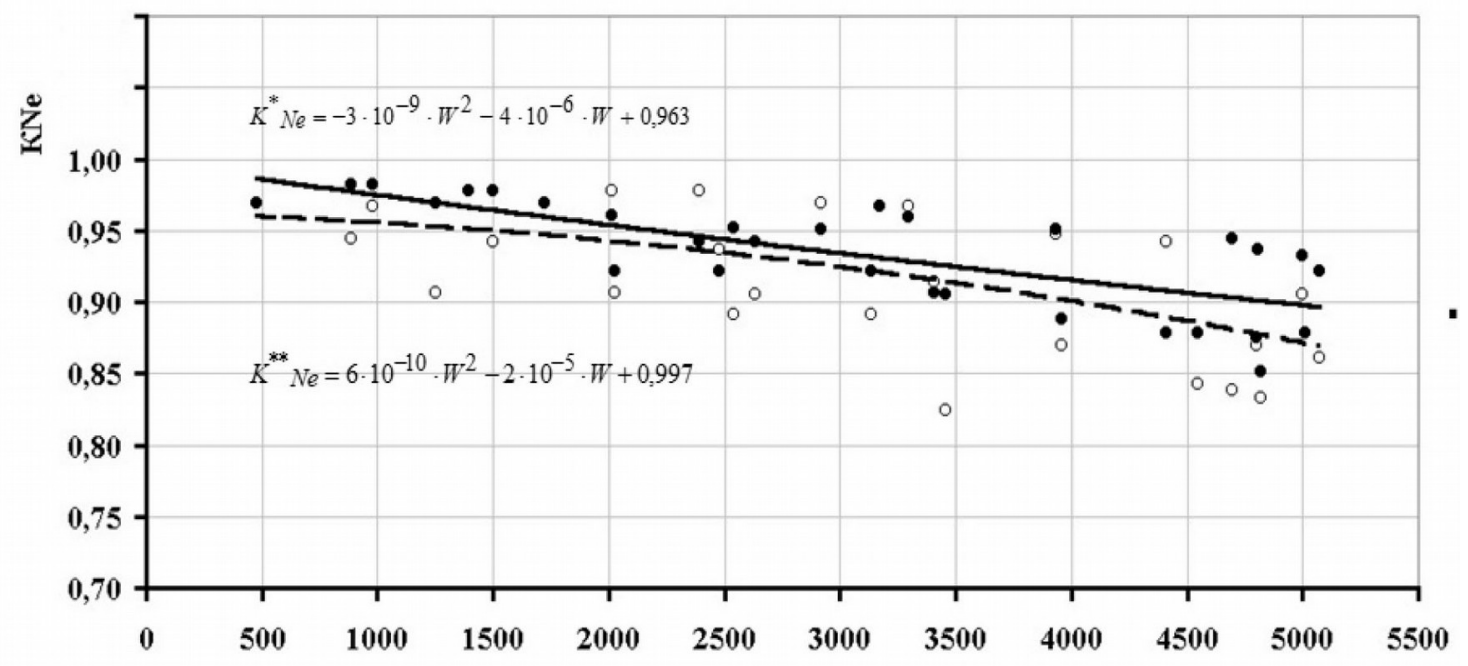



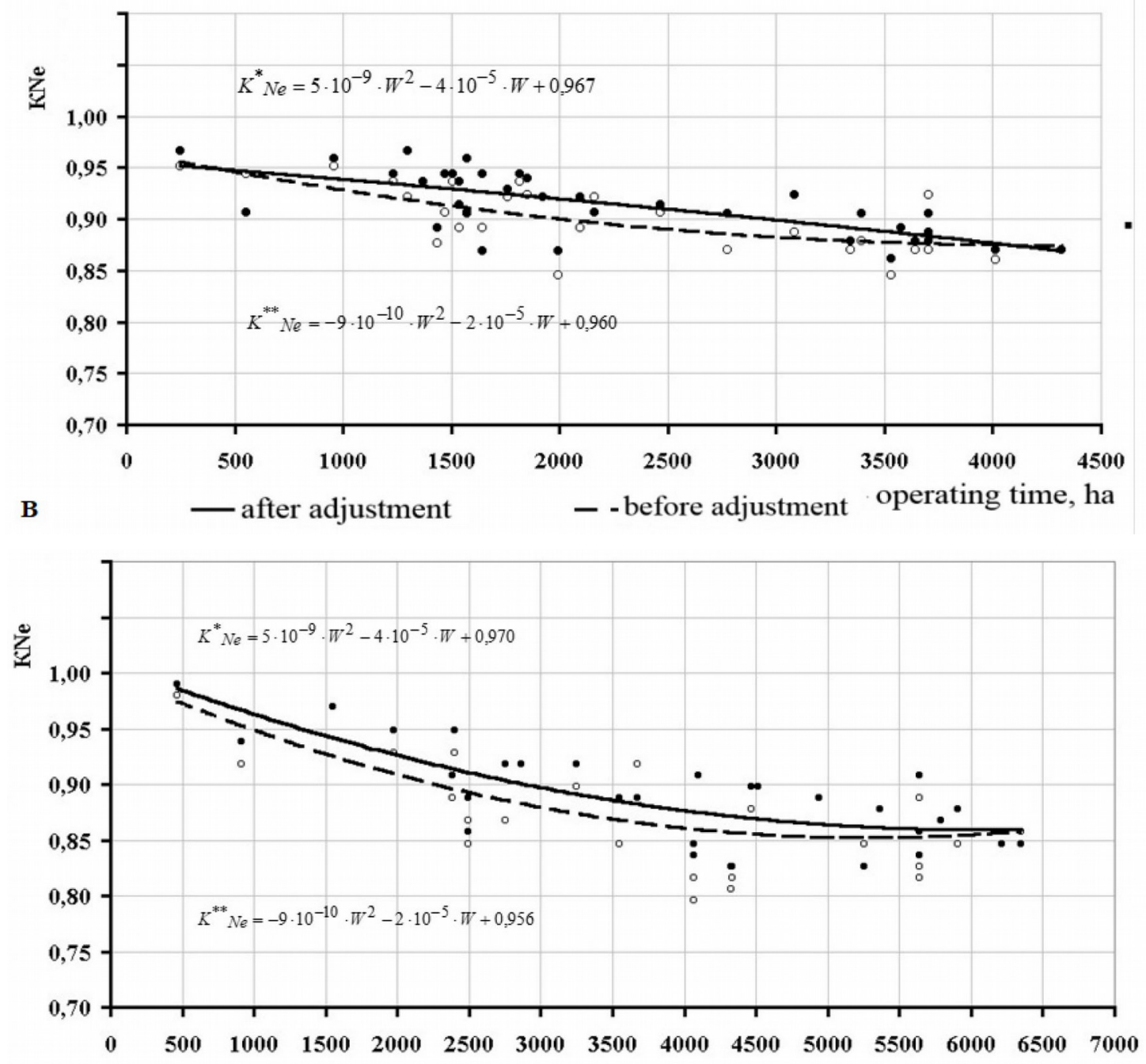

C - - before adjustment operating time, ha

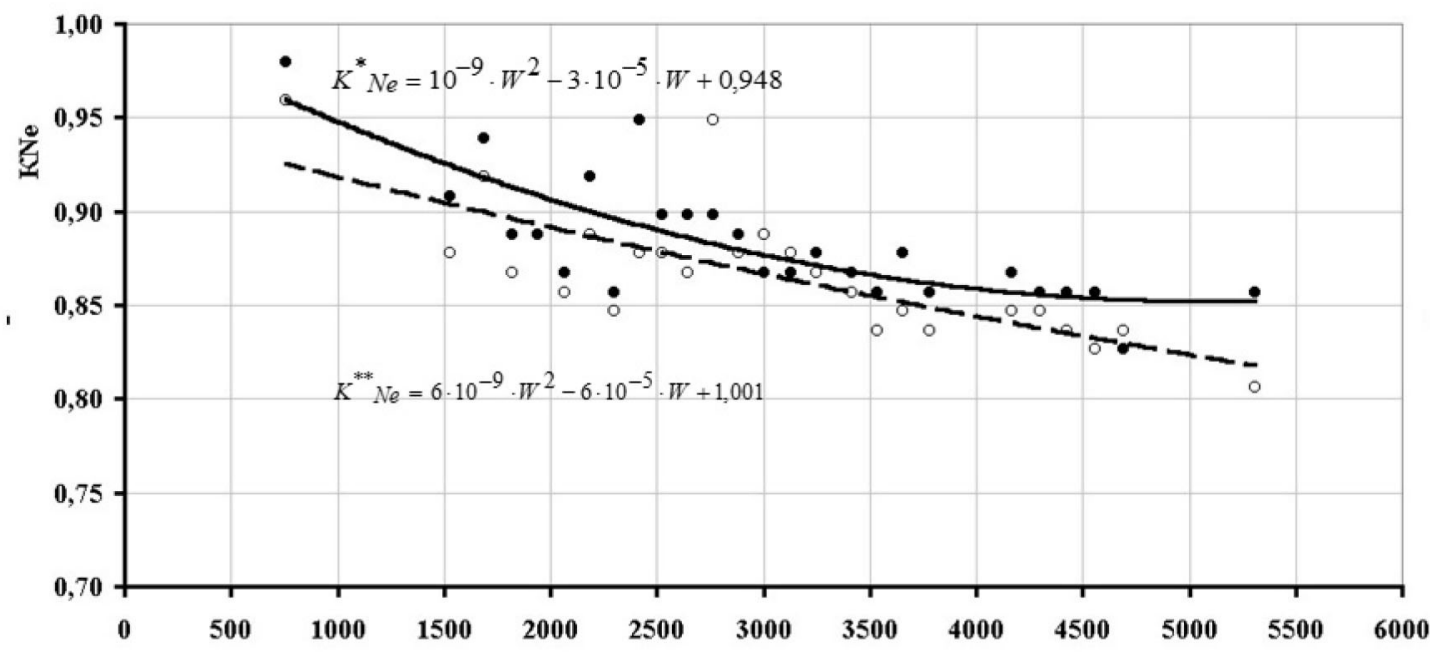

D $\quad$ - after adjustment

- -before adjustment operating time, ha

Figure 1. Dependence of the value of the coefficient $K_{N_{e}}$ on the operating time of tractors: a) $\Delta \mathrm{T}-75$ before overhaul; b) $\Delta \mathrm{T}-75$ after overhaul; c) T-402 before overhaul; d) T-402 after overhaul; *before adjustment; **after adjustment 
Second-order polynomial functions describe the dependencies shown in Figure 2. An analysis of the data shows that the engine power of the DT-75 brand tractors in the pre-repair period (corresponding to 4,000 ... 5,000 moto-hours of operation) falls by $8 \ldots 10 \%$, T-402 - by 13\%. After repair, the engine power is not fully restored and is $3 . .4 \%$ below the nominal value. The rate of power drop after the repair is 1.54 times higher than before the repair.

The engine power of the T-402 tractors decreases faster, and in the pre-repair period (3,500 ... 4,100 moto-hours) drops by $13 \%$. After repair, power decreases 1.41 times faster than before the repair.

Earlier studies found that among such indicators like age, work experience, and tractor driver class, the work experience has a decisive influence on the degree of fulfillment of the production norm. With this in mind, according to more than 100 observation sheets, the desired dependence was revealed (Figure 1).

A third-order polynomial function describes it $K W_{C M}=2 \times 10^{-5} S^{3}-0.0006 S^{2}+0,043 S+0.49$ the value of the multiple determination coefficient $\mathrm{R}^{2}$ is 0,9446 .

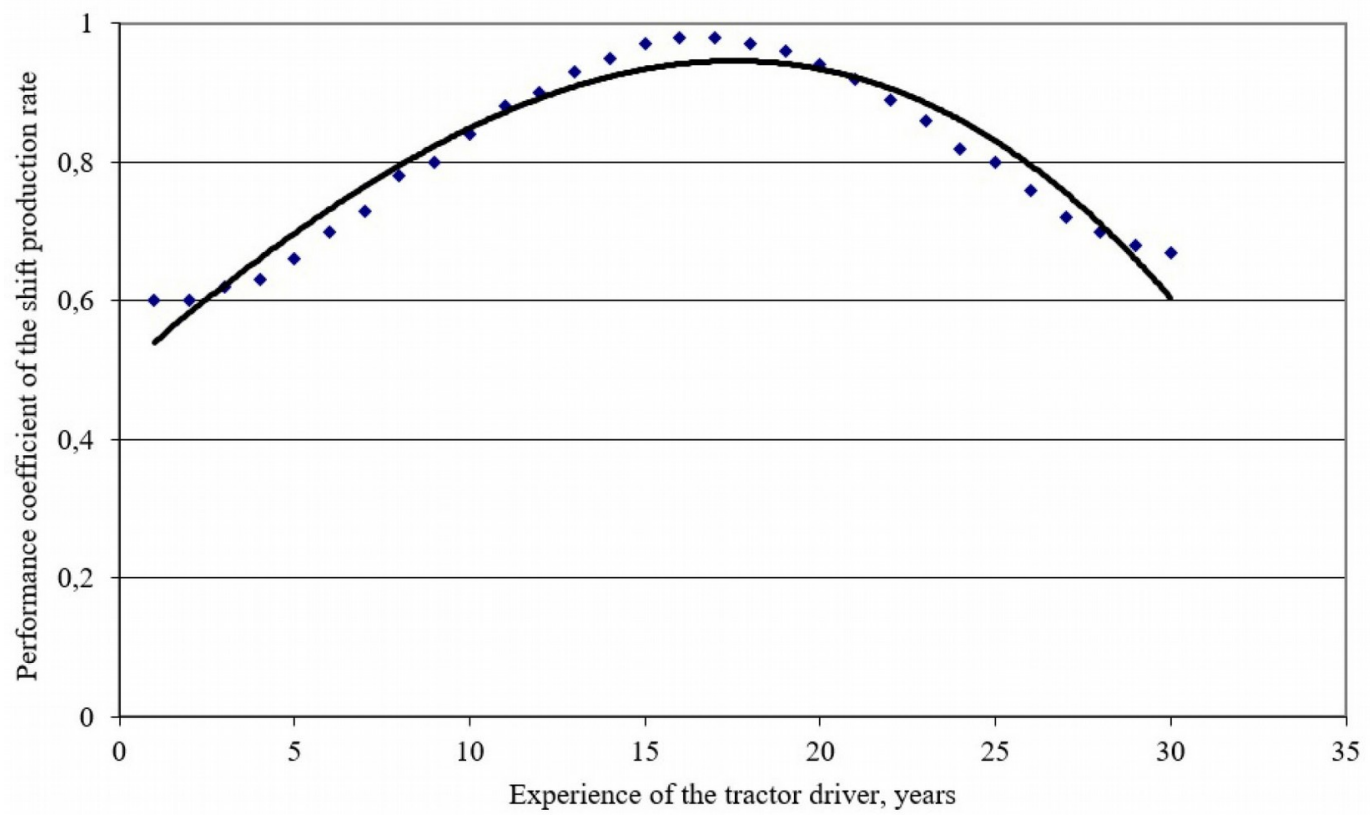

Figure 2. The dependence of the performance coefficient of the shift production rate on the experience of the tractor driver

As can be seen from Figure 2, in the first 1-3 years of work, the tractor operator, on average, fulfills the established production rate of $55-60 \%$. It is due to his inexperience. Also, young tractor drivers are most often given old tractors for work. That is, two equally directed factors contribute to a decrease in the value of this coefficient. The degree of fulfillment of the production norm increases and reaches a maximum with an experience of 15-16 years. But its value still rarely reaches $100 \%$. The norms are averaged for the entire economy. Also, they do not take into account the equipment "age", the tractor driver qualifications, and the field specific characteristics. A decrease in the degree of fulfillment of the norm of production is observed. It is due to the aging of the human body and rapid fatigue. Thus, the development of a 20 -year-old tractor driver (work experience $0-1$ years) is approximately equal to the production of 50-year-old one (experience 30 years).

The dependence of the value of their technical readiness of tractors coefficient on their service life was also investigated. It is known that the availability of equipment varies during the season, so it was studied in dynamics. Attempts to select equations to describe the desired dependencies using the classical technique of regression analysis, in particular, the least-squares method, were unsuccessful. 
In this regard, to solve the problem, neural network simulation was used. Note that previously, researchers did not use this relatively new type of modeling to solve such technical problems.

There are several main types of neural networks: multilayer perceptron (M.L.P.); radial basis functions (RBF), linear networks (Linear), generalized regression networks (G.R.N.N.). This indicator characterizes the completeness of their description of the desired dependence as the quality of the network (Performance). Among these types of networks, a network search was organized to solve the problem in the STATISTICA Neural Network 4.0 software environment. As a result of the search, a network of RBF type was found with the highest quality equal to 0.98 , which best describes the desired dependencies (Figure 3).

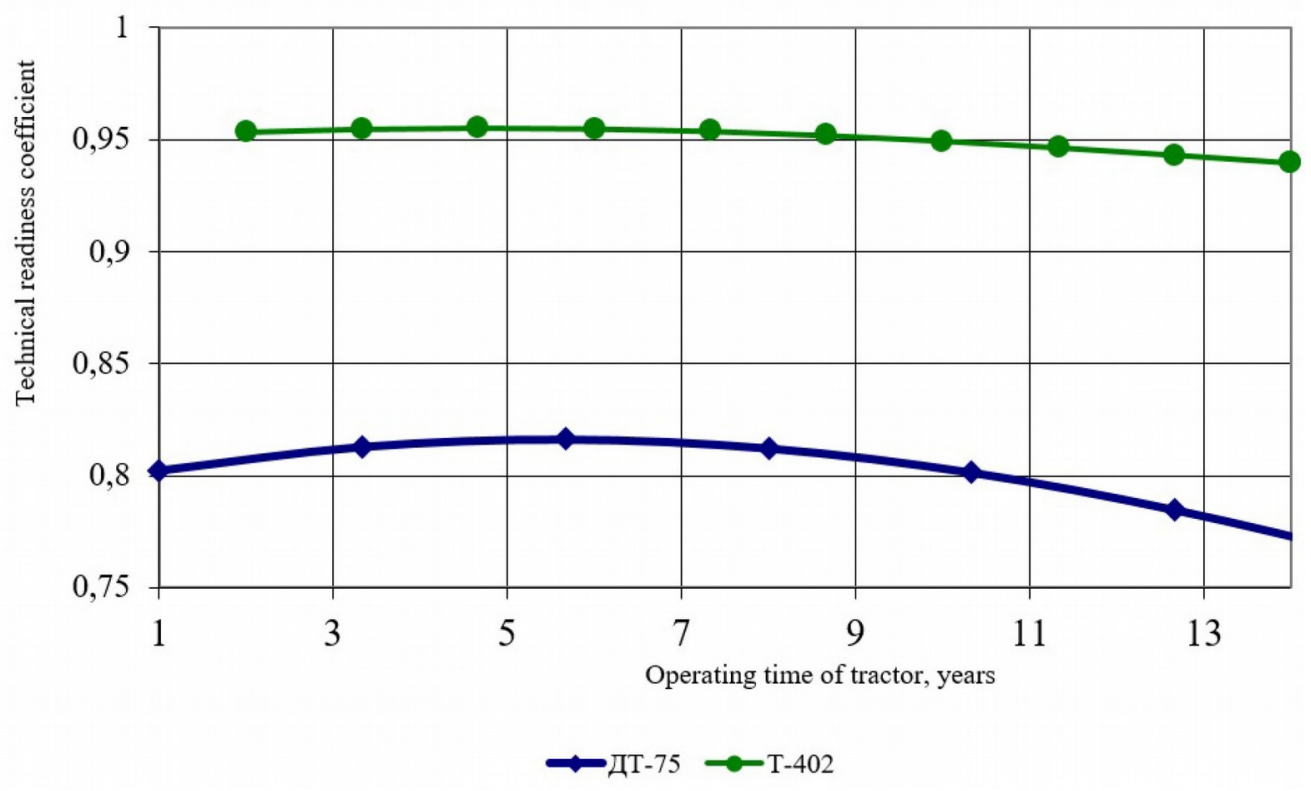

Figure 3. Dynamics of changes in the technical readiness of tractors (based on the results of neural network simulation)

From the graph (Figure 3), it can be seen that the value of the technical readiness coefficient of the studied tractors gradually increases and reaches a maximum by about 5-6 years of service. After that, it is steadily falling. For tractors of the T-402 brand (green line), it is the largest, compared to $\Delta \mathrm{T}-75$ (blue line). They are less idle due to technical malfunctions. Note that under ordinary operating conditions, the coefficient value does not reach unity. For $\mathrm{T}-402$ tractors, the maximum value reaches 0.9 , for $\Delta \mathrm{T}-75-0.86$.

\section{Discussion}

Optimization of the M.T.A. use plan is, by far, one of the most relevant and useful reserves for increasing the economic efficiency of agricultural production and without capital investments. The mathematical solution to this problem is often reduced to the traveling salesman problem (Wu et al., 2015a; Lin et al., 2016); elements of the theory of traffic flows are used (Parwanto et al., 2015). It is proposed to introduce additional blocks of separate temporal and spatial planning to take into account in the models the difficulties associated with the need for additional time to transfer the M.T.A. from one field to another and reaggregation (Wu et al., 2015b). A way to mathematically take into account the situation when the base of the territorial location of the equipment may not be the only one was proposed in Allate (2019). It is proposed to use different methods of linear programming, in particular, the "simplex method", taking into account the peculiarities of the statement of the resource allocation problem (Rüttimann, 2015; Tanaka, 2019), as well as nonlinear, dynamic, and integer programming. The latter method is the most preferable since the technique is integral units of the resource, and their values cannot be fractional. 
Despite the variety of models for the optimal distribution of M.T.A. by type of work, they do not take into account the "age" of the equipment, its technical readiness, the experience of the tractor driver, and often the current working conditions (Zainagabdinov, 2001). For planning in real production, this is unacceptable. If all these factors are taken into account, then it is necessary to distribute not the brands of tractors with an indication of their number, but each tractor individually. In this case, the available models of optimal planning for the use of M.T.A., as well as the mathematical apparatus, are not applicable in practice.

Given this, the authors developed a fundamentally new mathematical model for optimizing the use of technology, which, unlike existing ones, allows you to:

- plan the operation of each tractor individually;

- designate the so-called "mandatory tractors", that is, those that the user, at his discretion, would like to assign to a particular operation or vice versa would like the tractor operator not to work on this operation. It is especially important since, on the ground, the final decision on the use of technology is made subjectively. The developed model allows subjective corrections to be laid in advance before solving the problem and to obtain the optimal feasible plan.

To solve the problem, in addition to the model, the authors developed a mathematical apparatus for integer programming with Boolean variables based on the construction of the L.S. This method differs fundamentally from the existing ones by the ability to solve the problem without rounding the desired values. To construct an L.S., the authors derived a formula for calculating the value of any element.

Based on the results of experimental studies, functional dependencies for adjusting the performance of M.T.A. are identified, taking into account:

- "age" of the tractor based on the dynamics of its sufficient power;

- coefficient of technical readiness of the tractor;

- experience of a tractor driver.

It was established that the engines of tractors power of the $\triangle \mathrm{T}-75$ type in the pre-repair period $(4,000 \ldots 5,000$ moto-hours) fall by $8 \ldots 10 \%$. After a significant overhaul, the engine power is not fully restored and is $3 \ldots 4 \%$ lower than the nominal value. The rate of power drop after the overhaul is 1.54 times higher than before the repair.

The engine power of the T-402 tractors decreases faster, and in the pre-repair period (3,500... 4,100 moto-hours) drops by 13\%. After repair, power decreases 1.41 times faster than before the repair.

The restoration of operational adjustments makes it possible to increase the engines of $\Delta \mathrm{T}-75$ tractors power by an average of $2 \%$, and the T-402 tractors by 3\%. Moreover, after adjustments, the value of the coefficient of determination increases. It confirms the widespread prevalence of unauthorized regulation by the tractor drivers of the engine power system. In particular, we have recorded numerous cases of failure of seals of fuel equipment to increase the engine speed.

It is proved that M.T.A performance has a stronger correlation with the experience of the machine operator than with its class and age. A relationship between them is formalized, which has an extremum to take into account the influence of this factor on aggregate productivity. The maximum performance of the unit is achieved with the experience of the machine operator for 16 years.

For the first time, neural network modeling was used to study the dynamics of the tractor technical readiness coefficient, and the necessary functional dependencies were obtained.

Other researchers did not conduct experiments to obtain practically applicable functional dependencies for adjusting the performance of the M.T.A., taking into account the "age" of the equipment, its technical readiness, and the length of service of the tractor operator. The developed method and model of the optimal distribution of M.T.A. by type of work forms new branches in the tree of their classification.

Thus, the scientific novelty of the work performed is: 
- the technique for operational planning of work and increasing the efficiency of using the M.T.A., taking into account the technical condition of the tractors, the specific conditions for their functioning, and the length of service of the machine operator.

- the mathematical model for solving the problem of the optimal distribution of M.T.A. by type of work and the mathematical apparatus for its implementation;

- the technique for adjusting the performance of the M.T.A., taking into account the experience of the tractor driver, the service life, and the technical readiness of the tractor.

The developed mathematical model and analytical dependencies for adjusting the performance of M.T.A. were incorporated into the "AgroMaster+" computer program. Rospatent gave the certificate of official registration for the program. It allows in the conditions of a specific agricultural enterprise to quickly plan the operation of equipment. For practical testing, the program was introduced at the "Metevtamak" agricultural enterprise of the Republic of Bashkortostan. Operational distribution of tractors using the program on such simultaneously performed tasks as steam cultivation, continuous pre-sowing cultivation, winter rye sowing with mineral fertilizers, rolling crops, harvesting corn for silage, transporting silage yielded the following results:

- M.T.A. production rates adjusted according to the developed methodology are, on average equal to the actual production. They differ by no more than $10 \%$, while this difference between the standard rate and the actual output reaches $85 \%$;

- the use of technically sound standards as a factor in stimulating the labor of machine operators made it possible to increase the productivity of units by at least $7 \%$ and reduce fuel consumption by $10 \%$;

- due to optimal planning, the total duration of operations was reduced by 1.7 times compared to their actual duration. At the same time, the cost of additional products, calculated per 1 motor-hour, amounted to 7.3 dollars.

\section{Conclusions}

The developed economic and mathematical model, mathematical apparatus, and functional dependencies are implemented in the "AgroMaster+" computer program. The program allows in production conditions to calculate the actual possible production of units for specific conditions of their functioning, to draw up an implemented optimal plan for the use of MTP.

For the production verification of the research results, the "AgroMaster+" program was introduced at the "Metevtamak" agricultural enterprise of the Republic of Bashkortostan.

A production audit showed that the production rates corrected by the developed methodology are, on average equal to the actual production. They differ by no more than $10 \%$, while this difference between the standard rate and the actual output reaches $85 \%$.

The use of technically sound standards, being an essential factor in stimulating the work of machine operators, allows to increase the productivity of units by at least $7 \%$ and reduce fuel consumption by $10 \%$.

Due to optimal planning, the total duration of operations is reduced by 1.7 times compared to their actual duration. At the same time, the cost of additional products, calculated per 1 motor-hour, is 7.3 dollars.

Thus, the developed methodology for the optimal planning of the use of technology allows, without significant investment, to reduce the time of agricultural operations, reduce fuel consumption, costs in general, and thereby increase production efficiency. 


\section{Declaration of Conflicting Interests}

The authors declared no potential conflicts of interest with respect to the research, authorship, and/or publication of this article.

\section{Funding}

The authors received no financial support for the research, authorship, and/or publication of this article.

\section{References}

Allate, B.M. (2019). Terminal location models for intermodal transport network optimization. Open Journal of Applied Sciences, 9(05), 307. https://doi.org/10.4236/ojapps.2019.95025

Arzhenovskiy, A.G. (2017). Methods of determination of power and fuel-economic rates of machine and tractor units. Agricultural Machinery and Technologies, 6, 36-40. https://doi.org/10.22314/2073-7599-2017-6-36-40

Balas, E. (1967). Discrete programming by the filter method. Operations Research, 15(5), 915-957.

https://doi.org/10.1287/opre.15.5.915

Belahcene, S., Marthon, P., \& Aidene, M. (2018). The pivot adaptive method for solving linear programming problems. American Journal of Operations Research, 8(2), 92-111. https://doi.org/10.4236/ajor.2018.82008

Bibi, M.O., \& Bentobache, M. (2015). A hybrid direction algorithm for solving linear programs. International Journal of Computer Mathematics, 92(1), 201-216. https://doi.org/10.1080/00207160.2014.890188

Bulgakov, V., Kyurchev, V., Nadykto, V., Beloev, H., Kangalov, P., \& Mitev, G. (2017). Theoretical study to determine the standard size range of agricultural tractors. Mechanization in agriculture \& Conserving of the resources, 63(3), 92-94.

Jourquin, B. (2018). Estimating elasticities for freight transport using a network model: an applied methodological framework. Journal of Transportation Technologies, 9(01), 1. https://doi.org/10.4236/jtts.2019.91001

Lin, B.L., Sun, X., \& Salous, S. (2016). Solving travelling salesman problem with an improved hybrid genetic algorithm. Journal of computer and communications, 4(15), 98-106. https://doi.org/10.4236/jcc.2016.415009

Luo, Z. (2019). Path Selection of Multimodal Transport Based on Multi-Objective Mixed Integer Programming. Journal of Transportation Technologies, 9(4), 462-473. https://doi.org/10.4236/jtts.2019.94029

Parwanto, N.B., Morohosi, H., \& Oyama, T. (2015). Applying network flow optimization techniques to improve relief goods transport strategies under emergency situation. American Journal of Operations Research, 5(03), 95. https://doi.org/10.4236/ajor.2015.53009

Plaksin, A.M. (1975). Justification of the frequency of control and tolerance for engine power reduction during tractor operation in agriculture. Chelyabinsk.

Recht, P. (2016). A dynamic programming approach for the max-min cycle packing problem in even graphs. Open Journal of Discrete Mathematics, 6(4), 340-350. https://doi.org/10.4236/ojdm.2016.64027

Rüttimann, B.G. (2015). Discourse about linear programming and lean manufacturing: Two different approaches with a similar, converging rational. Journal of Service Science and Management, 8(01), 85.

https://doi.org/10.4236/jssm.2015.81010

Shevtsov, V., Lavrov, A., \& Izmailov, A. (2015). Formation of quantitative and age structure of tractorpark in the conditions of limitation of resources of agricultural production. SAE Technical Paper. https://doi.org/10.4271/2015-26-0147

Tanaka, Y. (2019). Using the simplex method for a type of allocation problems. American Journal of Computational Mathematics, 9(02), 25. https://doi.org/10.4236/ajcm.2019.92002

Wan, J., \& Wei, S. (2019). Research on route selection of multiobjective multimodal transport based on hybrid algorithm. Journal of Tianjin University (Natural Science and Engineering Tecbnology Edition), 52, 285-292. 
Wu, Y., Weise, T., \& Chiong, R. (2015). Local search for the traveling salesman problem: A comparative study. In 2015 IEEE 14th International Conference on Cognitive Informatics \& Cognitive Computing (ICCI* CC) (213-220). IEEE. https://doi.org/10.1109/ICCI-CC.2015.7259388

Wu, C., Zhou, L., Qiao, P., \& Wang, J. (2015). Addressing Uncertainty in Temporal and Spatial Scheduling for Farm Machinery Operation. Positioning, 7(1), 32-40. https://doi.org/10.4236/pos.2016.71003

Yakovenko, A., Makarchuk, V., \& Serbinov, V. (2019). Choice of the work of machine - tractor aggregates. Agrarian Bulletin of the Black Sea Littoria, 94, 117-121. https://doi.org/10.37000/abbsl.2019.94.17

Zainagabdinov, R.R. (2001). The Problems of Economic and Mathematical Models Using in a Machinery and Tractor Fleet Work Planning. In The 2-nd regional inter-university scientific and practical conference of young researchers and specialists with a presentation in foreign language (25-26). Ufa, B.S.A.U.

Zainagabdinov, R.R. (2004). Improving the efficiency of the use of machine-tractor units by improving their distribution by type of work, taking into account current operating conditions. St. Petersburg.

Zhao, W.J. (2017). Comparison of Technical and Economic Characteristics of Various Transportation Modes. Cooperative Economy and Technology, 3, 38-39.

Journal of Industrial Engineering and Management, 2020 (www.jiem.org)

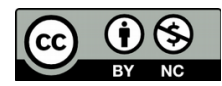

Article's contents are provided on an Attribution-Non Commercial 4.0 Creative commons International License. Readers are allowed to copy, distribute and communicate article's contents, provided the author's and Journal of Industrial Engineering and Management's names are included. It must not be used for commercial purposes. To see the complete license contents, please visit https://creativecommons.org/licenses/by-nc/4.0/. 